\title{
Systematic generation of entanglement measures for pure states
}

\section{Ayumu Sugita}

\begin{tabular}{|c|l|}
\hline Citation & Physical Review A. 77(5); 052304 \\
\hline Issue Date & 2008-05-02 \\
\hline Type & Journal Article \\
\hline Textversion & Publisher \\
\hline Rights & $\begin{array}{l}\text { C2008 American Physical Society. This article may be downloaded for personal } \\
\text { use only. Any other use requires prior permission of American Physical Society. }\end{array}$ \\
\hline DOI & 10.1103/PhysRevA.77.052304 \\
\hline Is Identical to & $\underline{\text { https://doi.org/10.1103/PhysRevA.77.052304 }}$ \\
\hline
\end{tabular}

Self-Archiving by Author(s)

Placed on: Osaka City University 


\title{
Systematic generation of entanglement measures for pure states
}

\author{
Ayumu Sugita* \\ Department of Applied Physics, Osaka City University, 3-3-138 Sugimoto, Sumiyoshi-ku, Osaka, 558-8585, Japan
}

(Received 28 September 2007; published 2 May 2008)

\begin{abstract}
We propose a method to generate entanglement measures systematically by using the irreducible decomposition of some copies of a state under the local unitary (LU) transformations. It is applicable to general multipartite systems. We show that there are entanglement monotones corresponding to singlet representations of the LU group. They can be evaluated efficiently in an algebraic way, and experimentally measurable by local projective measurements of the copies of the state. Nonsinglet representations are also shown to be useful to classify entanglement. Our method reproduces many well-known measures in a unified way.
\end{abstract}

DOI: 10.1103/PhysRevA.77.052304

PACS number(s): 03.67.Mn, 03.65.Fd

\section{INTRODUCTION}

Entanglement is one of the most striking features of quantum mechanics, and considered to be the key resource for quantum-information processing. In spite of the intensive study in the last decade, description and quantification of multipartite entanglement is still a challenging problem. A single quantity is not enough to characterize entanglement of multipartite states, since there can be qualitatively different quantum correlations. (A famous example is the Greenberger-Horne-Zeillinger (GHZ) state and $W$ states in a 3-qubit system [1].) Therefore we need more than one measure to classify multipartite entanglement, and the number of the necessary measures grows rapidly as the number of parties increases.

An important requirement for entanglement measure is to be entanglement monotone, i.e., nonincreasing under stochastic local operations and classical communication (SLOCC). So far, constructions of entanglement monotones have been made mostly in heuristic ways using specific features of the system under consideration. To the best of the author's knowledge, the only method of construction of entanglement monotones which is applicable to general multipartite system is the hyperdeterminant [2]. However, we can make only one entanglement monotone for a system from the hyperdeterminant. Furthermore, the polynomial degree of the hyperdeterminant grows very fast as the number of the parties increases, which makes it difficult to write down its explicit form. Therefore we need a method to generate many entanglement monotones for an arbitrary multipartite system, hopefully in a systematic and efficient way.

In this paper, we propose a method which satisfies all these requirements. We consider $q$ copies of a state $\left|\psi^{\otimes q}\right\rangle$, where $q$ is an arbitrary positive integer, and decompose it into irreducible components under the LU group. Then the norm of an irreducible component can be regarded as a measure of entanglement. Its explicit form is given by the Clebsch-Gordan coefficients of the LU group and the expansion coefficients of the given state. Our method enables us to make a list of all algebraic invariants of the LU group of a given order.

\footnotetext{
*sugita@a-phys.eng.osaka-cu.ac.jp
}

Irreducible decomposition of $\left|\psi^{\otimes q}\right\rangle$ was first introduced in [3] in the context of algebraic evaluation of the moments of the generalized Husimi distribution. Our method can be regarded as a generalization of the definition of the concurrence for multipartite systems in [4], in which irreducible decomposition of $\left|\psi^{\otimes 2}\right\rangle$ was considered. Our method also includes the construction of entanglement monotones for multiqubit states in [5] with use of expectation values of antilinear operators. The expectation value of an antilinear operator $A$ is written as $\langle A\rangle=\langle\psi|L C| \psi\rangle$, where $L$ is a linear operator and $C$ is the complex conjugation. If we expand the state as $|\psi\rangle=\Sigma_{j} c_{j}|j\rangle$, the expectation value can be written explicitly as $\Sigma_{i, j}=L_{i, j} c_{i}^{*} c_{j}^{*}$, where $L_{i, j}=\langle i|L| j\rangle$. Then $\langle A\rangle^{*}$ $=\sum_{i, j} L_{i, j} c_{i} c_{j}$ is considered as a linear map from $\left|\psi^{\otimes 2}\right\rangle$ to a complex number. Therefore if $|\langle A\rangle|$ is invariant under the LU group, it must be obtained from a singlet component of $\left|\psi^{\otimes 2}\right\rangle$ [6].

The purpose of this paper is to show that our group theoretical method gives a quite general and unified point of view for multipartite entanglement measures. Therefore, in the following, we concentrate mainly on describing the general theory and deriving well-known existing measures from our method. Analysis of complicated entanglement in specific systems will be reported elsewhere.

\section{DESCRIPTION OF THE GENERAL METHOD}

First we describe our method as generally as possible. Let us consider an $m$-partite system with $N_{i}$ states for $i$ th party. The LU group of this system is $\mathrm{SU}\left(N_{1}\right)$ $\times \mathrm{SU}\left(N_{2}\right) \times \cdots \times \mathrm{SU}\left(N_{m}\right)$. Since an irreducible representation (irrep) of a special unitary group is specified by its highest weight [7], we denote an irrep with the highest weight $\nu$ by $R_{\nu}$. Then an irreducible representation of the LU group can be written as $R_{\nu_{1}} \otimes R_{\nu_{2}} \otimes \cdots \otimes R_{\nu_{m}}$, where $R_{\nu_{i}}$ is the irreducible representation of $\mathrm{SU}\left(N_{i}\right)$ with the highest weight $\nu_{i}$. We denote this representation by $R_{\boldsymbol{\nu}}$, where the bold symbol $\boldsymbol{\nu} \equiv\left(\nu_{1}, \nu_{2}, \ldots, \nu_{m}\right)$ represents the set of the highest weights. A purestate $|\psi\rangle$ in this system is in the defining representation of the LU group, which is denoted by $R_{\boldsymbol{e}}$. Here, $\boldsymbol{e}$ $\equiv\left(e_{1}, e_{2}, \ldots, e_{m}\right)$, and $e_{i}$ is the highest weight of the defining representation of $\mathrm{SU}\left(N_{i}\right)$. Explicit form of the highest weight depends on the choice of the basis of the Lie algebra. For 
example, in the convention of Ref. [7], $e_{i}$ $=\left(\frac{1}{2}, \frac{1}{2 \sqrt{3}}, \ldots, \frac{1}{\sqrt{2 m(m+1)}}, \ldots, \frac{1}{\sqrt{2\left(N_{i}-1\right) N_{i}}}\right)$.

Then we consider $q$ copies of a state $\left.\left|\psi^{\otimes q}\right\rangle \equiv|\psi\rangle\right\rangle^{\otimes q}$. Since $R_{e}^{\otimes q}$ is reducible for $q \geq 2$, we can decompose it into irreducible components as

$$
R_{e}^{\otimes q}=\oplus_{\alpha} R_{\nu_{\alpha}} .
$$

$\left|\psi^{\otimes q}\right\rangle$ is decomposed correspondingly as

$$
\left|\phi^{\otimes q}\right\rangle=\sum_{\alpha} P_{\nu_{\alpha}}\left|\phi^{\otimes q}\right\rangle
$$

where $P_{\nu_{\alpha}} \equiv P_{\nu_{\alpha, 1}} \otimes \cdots \otimes P_{\nu_{\alpha, m}}$ is the projection operator to the representation space of $R_{\boldsymbol{\nu}_{\alpha}}^{\alpha, m}$. Among the irreducible components, there is always a unique component with the "maximum" highest weight $q \boldsymbol{e}=\left(q e_{1}, \ldots, q e_{m}\right)$. It has been shown in [8] that $\left|\psi^{\otimes q}\right\rangle \in R_{q e}$ iff $|\psi\rangle$ is not entangled. Therefore, for $q \geq 2$, we can conclude that

$$
\left.|\psi\rangle \text { is unentangled } \Leftrightarrow\left|P_{q e}\right| \psi^{\otimes q}\right\rangle \mid=1
$$

assuming $|\psi\rangle$ is normalized, and

$$
|\psi\rangle \text { is unentangled } \Rightarrow P_{\nu_{\alpha}}\left|\psi^{\otimes q}\right\rangle=0
$$

for $\boldsymbol{\nu}_{\alpha} \neq q \boldsymbol{e}$.

It has been shown that $\left.\left|P_{q e}\right| \psi^{\otimes q}\right\rangle\left.\right|^{2}$ is the $q$ th moment of a generalized Husimi distribution up to a constant multiplier $[3,8]$, and the Rényi subentropy defined from the moment is an entanglement monotone for $N \times N$ bipartite systems [9].

$\left.\left|P_{\nu_{\alpha}}\right| \psi^{\otimes q}\right\rangle \mid$ with $\boldsymbol{\nu}_{\alpha} \neq q \boldsymbol{e}$ is also a good candidate measure of entanglement since it is invariant under LU transformations and vanishes for unentangled states. Singlet representations, which have the "minimum" highest weight $\boldsymbol{\nu}_{\alpha}=\mathbf{0}$ $\equiv(0, \ldots 0)$, are of particular interest. (Note that 0 within the bracket is not a number, but the zero weight vector.) Actually we can show that $\left.\left|P_{\boldsymbol{\nu}_{\alpha}=0}\right| \psi^{\otimes q}\right\rangle\left.\right|^{1 / q}$ is an entanglement monotone.

Proof of monotonicity. We use a theorem proved in [10] which claims that a linearly homogeneous positive function of a pure state that remains invariant under determinant 1 SLOCC operations is an entanglement monotone. Since it is obvious that $\left.\left|P_{\boldsymbol{\nu}_{\alpha}=\mathbf{0}}\right| \psi^{\otimes q}\right\rangle\left.\right|^{1 / q}$ is linearly homogeneous and positive, what we have to show is its invariance under the SLOCC group $S L\left(N_{1}, \mathrm{C}\right) \times \cdots \times S L\left(N_{m}, \mathrm{C}\right)$.

If we consider a singlet representation $R_{\nu=0}$ of $\mathrm{SU}(N)$, it is obvious that $T|\phi\rangle=0$ for any $T \in \mathfrak{s} u(N)$ and $|\phi\rangle \in R_{\nu=0}$, where the fracture letters denote the Lie algebra of the corresponding Lie group. Since $\mathfrak{s l}(N, \mathrm{C})$ is obtained as the complexification of $\mathfrak{s} u(N)$, i.e., the set of linear combinations of its elements with complex coefficients, $T|\phi\rangle=0$ holds also for $\forall T \in \mathfrak{s l}(N, \mathrm{C})$. Therefore an element of the singlet representation of $\operatorname{SU}(N)$ is also invariant under $\operatorname{SL}(N, \mathrm{C})$. In the same way, $P_{\boldsymbol{\nu}_{\alpha}=0}\left|\psi^{\otimes q}\right\rangle$ is shown to be invariant under $\operatorname{SL}\left(N_{1}, \mathrm{C}\right) \times \cdots \times \operatorname{SL}\left(N_{m}, \mathrm{C}\right)$.

\section{2-QUBIT CASE}

Let us consider a 2-qubit system to see how our general theory works. A qubit is in the defining (spin 1/2) represen- tation of $\mathrm{SU}(2)$, which is $R_{1 / 2}$ in our notation. Note that the highest weight is the total spin quantum number in this case. In general, the tensor product of two irreducible representations of $\mathrm{SU}(2)$ is decomposed as $R_{m} \otimes R_{n}=R_{m+n} \oplus R_{m+n-1}$ $\oplus \cdots \oplus R_{|m-n|}$.

In the simplest case $q=2$, two copies of a qubit is decomposed into a triplet $R_{1}$ and a singlet $R_{0}$. Hereafter we arrange the tensor product to represent copies of a quantum state vertically in order to distinguish it from the tensor product to represent multipartite states. Then the irreducible decomposition of the two copies of a qubit can be written as

$$
R_{1 / 2}^{\otimes 2}=\underset{R_{1 / 2}}{R_{1 / 2}}=R_{1} \oplus R_{0} .
$$

Since a 2-qubit state is in the representation $R_{1 / 2} \otimes R_{1 / 2}$, two copies of a 2-qubit state is decomposed as

$$
\begin{aligned}
\left(R_{1 / 2} \otimes R_{1 / 2}\right) & \otimes\left(\begin{array}{c}
R_{1 / 2} \\
\otimes \\
\left(R_{1 / 2} \otimes R_{1 / 2}\right)
\end{array}\right) \otimes\left(\begin{array}{c}
R_{1 / 2} \\
R_{1 / 2}
\end{array}\right) \\
& =\left(R_{1} \oplus R_{0}\right) \otimes\left(R_{1} \oplus R_{0}\right) \\
& =R_{1} \otimes R_{1} \oplus R_{1} \otimes R_{0} \oplus R_{0} \otimes R_{1} \oplus R_{0} \otimes R_{0} .
\end{aligned}
$$

Here, $R_{1} \otimes R_{0}$ and $R_{0} \otimes R_{1}$ are antisymmetric with respect to the exchange of the two copies, because $R_{1}$ is symmetric and $R_{0}$ is antisymmetric. Since $\left|\psi^{\otimes 2}\right\rangle$ is symmetric, $P_{1}$ $\otimes P_{0}\left|\psi^{\otimes 2}\right\rangle$ and $P_{0} \otimes P_{1}\left|\psi^{\otimes 2}\right\rangle$ vanish identically. Hence the irreducible decomposition of the two copies of a 2-qubit state is

$$
\left|\psi^{\otimes 2}\right\rangle=P_{1} \otimes P_{1}\left|\psi^{\otimes 2}\right\rangle+P_{0} \otimes P_{0}\left|\psi^{\otimes 2}\right\rangle .
$$

The squared norm of the first term is the second moment of the generalized Husimi distribution $[3,11]$ up to a constant multiplier. The second term is the projection to the singlet representation $R_{0} \otimes R_{0}$. Hence the square root of its norm is an entanglement monotone.

Let us derive the explicit form of $P_{0} \otimes P_{0}\left|\psi^{\otimes 2}\right\rangle$. The basis vector of the second order singlet $R_{0}$ is

$$
\begin{aligned}
\left|s_{2}\right\rangle & \left.\equiv \frac{1}{\sqrt{2}}\left(\begin{array}{l}
0 \\
1
\end{array}\right\rangle-\left|\begin{array}{l}
1 \\
0
\end{array}\right\rangle\right) \\
& \left.=\frac{\epsilon_{i j}}{\sqrt{2}} \mid \begin{array}{l}
i \\
j
\end{array}\right),
\end{aligned}
$$

where $\epsilon_{i j}$ is the completely antisymmetric tensor. Note that the Einstein summation convention is used and the indices take on the values 0 or 1 . Then the basis vector of $R_{0} \otimes R_{0}$ is

$$
\left|s_{2}\right\rangle \otimes\left|s_{2}\right\rangle
$$

$$
=\frac{1}{2} \epsilon_{i_{11} i_{21}} \epsilon_{i_{12} i_{22}}\left|\begin{array}{ll}
i_{11} & i_{12} \\
i_{21} & i_{22}
\end{array}\right\rangle
$$




$$
=\frac{1}{2}\left(\left|\begin{array}{ll}
0 & 0 \\
1 & 1
\end{array}\right\rangle+\left|\begin{array}{ll}
1 & 1 \\
0 & 0
\end{array}\right\rangle-\left|\begin{array}{ll}
0 & 1 \\
1 & 0
\end{array}\right\rangle-\left|\begin{array}{ll}
1 & 0 \\
0 & 1
\end{array}\right\rangle\right) .
$$

Note that the set of indices $\left\{i_{j k}\right\}$ forms a $q \times m$ matrix in general. We expand the state $|\psi\rangle$ in the standard basis as $|\psi\rangle=c_{i j}|i j\rangle$. Then

$$
\begin{aligned}
& c_{i j}|i j\rangle \\
& \left|\psi^{\otimes 2}\right\rangle=\otimes \\
& c_{k l}|k l\rangle \\
& =c_{i_{11} i_{12}} c_{i_{21} i_{22}}\left|\begin{array}{ll}
i_{11} & i_{12} \\
i_{21} & i_{22}
\end{array}\right\rangle .
\end{aligned}
$$

Therefore

$$
\begin{aligned}
\left.\left|P_{0} \otimes P_{0}\right| \psi^{\otimes 2}\right\rangle \mid & =\mid\left(\left\langle s_{2}\left|\otimes\left\langle s_{2}\right|\right) \mid \psi^{\otimes 2}\right\rangle \mid\right. \\
& =\frac{1}{2}\left|\epsilon_{i_{11} i_{21}} \epsilon_{i_{12} i_{22}} c_{i_{11} i_{12}} c_{i_{21} i_{22}}\right| \\
& =\left|c_{11} c_{00}-c_{10} c_{01}\right| .
\end{aligned}
$$

This is the concurrence for pure states except for a factor of two.

\section{3-QUBIT CASE}

Next we consider a 3-qubit system. For $q=2$, we have a singlet $\left|s_{2}\right\rangle \otimes\left|s_{2}\right\rangle \otimes\left|s_{2}\right\rangle$ but this is antisymmetric with respect to the exchange of the two copies. Therefore the projection of $\left|\psi^{\otimes 2}\right\rangle$, where $|\psi\rangle$ is a 3 -qubit state, to this component vanishes identically.

Then we have to consider larger $q$ to find nontrivial entanglement monotones. Since it is impossible to make a singlet from three copies a qubit, the next candidate is $q=4$. In this case, the irreducible decomposition is

$$
R_{1 / 2}^{\otimes 4}=\left(R_{1} \oplus R_{0}\right)^{\otimes 2}=R_{2} \oplus 3 R_{1} \oplus 2 R_{0} .
$$

Therefore we can make two different singlets. One is included in the combination of the two triplets $R_{1}^{\otimes 2}=R_{2} \oplus R_{1}$ $\oplus R_{0}$. Its explicit form is

$$
\begin{aligned}
\left|s_{4, a}\right\rangle \equiv & \left.\left.\frac{1}{2 \sqrt{3}}\left(\begin{array}{l}
0 \\
0 \\
1 \\
1
\end{array}\right)+2 \mid \begin{array}{l}
1 \\
1 \\
0 \\
0
\end{array}\right)-\mid \begin{array}{l}
0 \\
1 \\
0 \\
1
\end{array}\right) \\
& \left.\left.-\left|\begin{array}{l}
1 \\
0 \\
1 \\
0
\end{array}\right|-\mid \begin{array}{l}
0 \\
1 \\
1 \\
0
\end{array}\right)-\left|\begin{array}{l}
1 \\
0 \\
0 \\
1
\end{array}\right|\right) \\
& =d_{i j k l}\left|\begin{array}{l}
i \\
j \\
k \\
l
\end{array}\right\rangle,
\end{aligned}
$$

where

$$
d_{i j k l} \equiv \begin{cases}\frac{1}{\sqrt{3}} & {[(i j k l)=(1100),(0011)]} \\ -\frac{1}{2 \sqrt{3}} & {[(i j k l)=(1010),(0101),(1001),(0110)]} \\ 0 & \text { else }\end{cases}
$$

The other singlet is $\left|s_{4, b}\right\rangle \equiv\left|s_{2}\right\rangle^{\otimes 2}$, i.e., two copies of the second order singlet $\left|s_{2}\right\rangle$. It can be written explicitly as

$$
\begin{aligned}
\left|s_{4, b}\right\rangle & =\frac{1}{2} \epsilon_{i j} \epsilon_{k l}\left|\begin{array}{l}
i \\
j \\
k \\
l
\end{array}\right| \\
& \left.=\frac{1}{2}\left(\left|\begin{array}{l}
0 \\
1 \\
0 \\
1
\end{array}\right|+\mid \begin{array}{l}
1 \\
0 \\
1 \\
0
\end{array}\right)-\left|\begin{array}{l}
0 \\
1 \\
1 \\
0
\end{array}\right|-\left|\begin{array}{l}
1 \\
0 \\
0 \\
1
\end{array}\right|\right) .
\end{aligned}
$$

We can make entanglement monotones by using these singlets. For example, $\left|s_{4, a}\right\rangle \otimes\left|s_{4, a}\right\rangle \otimes\left|s_{4, a}\right\rangle$ is represented as

$$
\begin{aligned}
& \left|s_{4, a}\right\rangle \otimes\left|s_{4, a}\right\rangle \otimes\left|s_{4, a}\right\rangle \\
& =d_{i_{11} i_{21} i_{31} i_{41}} d_{i_{12} i_{22} i_{32} i_{42}} d_{i_{13} i_{23} i_{33} i_{43}}\left|\begin{array}{lll}
i_{11} & i_{12} & i_{13} \\
i_{21} & i_{22} & i_{23} \\
i_{31} & i_{32} & i_{33} \\
i_{41} & i_{42} & i_{43}
\end{array}\right|,
\end{aligned}
$$

and

$$
\left|\psi^{\otimes 4}\right\rangle=c_{i_{11} i_{12} i_{13}} c_{i_{21} i_{22} i_{23}} c_{i_{31} i_{32} i_{33}} c_{i_{41} i_{42} i_{43}}\left|\begin{array}{ccc}
i_{11} & i_{12} & i_{13} \\
i_{21} & i_{22} & i_{23} \\
i_{31} & i_{32} & i_{33} \\
i_{41} & i_{42} & i_{43}
\end{array}\right|,
$$

where $|\psi\rangle=c_{i j k}|i j k\rangle$ is a 3 -qubit state. Then we obtain

$$
\begin{aligned}
\mid\left(\left\langle s_{4, a}\right| \otimes\right. & \left\langle s_{4, a}\left|\otimes\left\langle s_{4, a}\right|\right) \mid \psi^{\otimes 4}\right\rangle \mid \\
= & \mid d_{i_{11} i_{21} i_{31} i_{41}} d_{i_{12} i_{22} i_{32} i_{42}} d_{i_{13} i_{23} i_{33} i_{43}} \\
& \times c_{i_{11} i_{12} i_{13}} c_{i_{21} i_{22} i_{23}} c_{i_{31} i_{32} i_{33} c_{41} c_{i_{42} i_{43}} \mid .}
\end{aligned}
$$

By explicit calculation it is shown to be $\frac{\tau_{3}}{8 \sqrt{3}}$, where $\tau_{3}$ is the 3-tangle [12].

There are other fourth order singlets: $\left|s_{4, b}\right\rangle \otimes\left|s_{4, a}\right\rangle \otimes\left|s_{4, a}\right\rangle$, $\left|s_{4, b}\right\rangle \otimes\left|s_{4, b}\right\rangle \otimes\left|s_{4, a}\right\rangle,\left|s_{4, b}\right\rangle \otimes\left|s_{4, b}\right\rangle \otimes\left|s_{4, b}\right\rangle$ and their permutations. However, we have to use singlets with even number of $\left|s_{4, b}\right\rangle$, since $\left|s_{4, b}\right\rangle$ is antisymmetric with respect to the exchange of a pair of copies of $|\psi\rangle$. Therefore we use $\left|s_{4, b}\right\rangle$ $\otimes\left|s_{4, b}\right\rangle \otimes\left|s_{4, a}\right\rangle$, and obtain 


$$
\begin{aligned}
&\left(\left\langle s_{4, b}\right| \otimes\left\langle s_{4, b}\left|\otimes\left\langle s_{4, a}\right|\right) \mid \psi^{\otimes 4}\right\rangle\right. \\
&=\frac{1}{4} \mid \epsilon_{i_{11} i_{21}} \epsilon_{i_{31} i_{41}} \epsilon_{i_{12} i_{22}} \epsilon_{i_{32} i_{42}} d_{i_{13} i_{23} i_{33} i_{43}} \\
& \times c_{i_{11} i_{12} i_{13}} c_{i_{21} i_{22} i_{23}} c_{i_{31} i_{32} i_{33}} c_{i_{41} i_{42} i_{43}} \mid \\
&= \frac{\tau_{3}}{8 \sqrt{3}} .
\end{aligned}
$$

Considering the permutation symmetry of $\tau_{3}$, we see that $\tau_{3}$ is the only fourth-order invariant polynomial in the 3 -qubit case.

It is possible to choose another basis for the fourth order singlets. For example, let us make two second order singlets by combining the first and third copies, and the second and the fourth ones. By putting together the two second order singlets we obtain another fourth order singlet

$$
\begin{aligned}
\left|s_{4, c}\right\rangle & =\frac{1}{2} \epsilon_{i k} \epsilon_{j l}\left|\begin{array}{l}
i \\
j \\
k \\
l
\end{array}\right| \\
& \left.\left.=\frac{1}{2}\left(\mid \begin{array}{l}
0 \\
0 \\
1 \\
1
\end{array}\right)+\mid \begin{array}{l}
1 \\
1 \\
0 \\
0
\end{array}\right)-\mid \begin{array}{l}
1 \\
0 \\
0 \\
1
\end{array}\right)-\left(\begin{array}{l}
0 \\
1 \\
1 \\
0
\end{array} \mid\right)
\end{aligned}
$$

It is linearly dependent on $\left|s_{4, a}\right\rangle$ and $\left|s_{4, b}\right\rangle:\left|s_{4, c}\right\rangle=\frac{\sqrt{3}}{2}\left|s_{4, a}\right\rangle$ $+\frac{1}{2}\left|s_{4, b}\right\rangle$. Therefore it does not give any new measure, but it gives new expressions for $\tau_{3}$. For example,

$$
\begin{aligned}
&\left(\left\langle s_{4, b}\right| \otimes\left\langle s_{4, b}\left|\otimes\left\langle s_{4, c}\right|\right) \mid \psi^{\otimes 4}\right\rangle\right. \\
&=\frac{1}{8} \mid \epsilon_{i_{11} i_{21}} \epsilon_{i_{31} i_{41}} \epsilon_{i_{12} i_{22}} \epsilon_{i_{32} i_{42}} \epsilon_{i_{13} i_{33}} \epsilon_{i_{23} i_{43}} \\
& \times c_{i_{11} i_{12} i_{13}} c_{i_{21} i_{22} i_{23}} c_{i_{31} i_{32} i_{33}} c_{i_{41} i_{42} i_{43}} \mid \\
&= \frac{\tau_{3}}{16}
\end{aligned}
$$

is the original form of the 3-tangle given in [12].

\section{OTHER CASES}

We can construct entanglement monotones for multiqubit systems in the same way. The simplest singlet is $\left|s_{2}\right\rangle \otimes \cdots$ $\otimes\left|s_{2}\right\rangle$. The corresponding entanglement measure is

$$
\frac{1}{2^{m / 2}}\left|\epsilon_{i_{11} i_{21}} \epsilon_{i_{12} i_{22}} \ldots \epsilon_{i_{1 m} i_{2 m}} c_{i_{11} i_{12} \ldots i_{1 m}} c_{i_{21} i_{22} \ldots i_{2 m}}\right|,
$$

which is a generalization of the concurrence. Although this second order measure vanishes identically for odd $m$, we can construct fourth order measures using $\left|s_{4, a}\right\rangle$ and $\left|s_{4, b}\right\rangle$. For example, the measure corresponding to $\left|s_{4, a}\right\rangle \otimes \cdots \otimes\left|s_{4, a}\right\rangle$ is

$$
\left|d_{i_{11} i_{21} i_{31} i_{41}} \ldots d_{i_{1 m} i_{2 m} i_{3 m} i_{4 m}} c_{i_{11} \ldots i_{1 m}} c_{i_{21} \ldots i_{2 m}} c_{i_{31} \ldots i_{3 m}} c_{i_{41} \ldots i_{4 m}}\right|,
$$

which is a natural generalization of the 3-tangle. It vanishes for the $m$-qubit $W$ state $\left|W_{m}\right\rangle$, since $\left|W_{m}\right\rangle^{\otimes 4}$ is an eigenstate of the $z$ component of the total spin with eigenvalue $2 m$, and hence has no singlet component. For the $m$-qubit GHZ state, it takes the value $\left\{2^{m+1}+4(-1)^{m}\right\} /(2 \sqrt{3})^{m}$. Unlike the 3 -qubit case, there are many other fourth order invariants for $m \geq 4$.

It is also possible to construct entanglement measures for multilevel systems. As a simple example, let us consider a pair of qutrits. The simplest SU(3) singlet which can be constructed from qutrits is the third order completely antisymmetric state

$$
\left|s_{3}\right\rangle \equiv \frac{1}{\sqrt{6}} \epsilon_{i j k}\left|\begin{array}{l}
i \\
j \\
k
\end{array}\right| .
$$

Note that the indices take on one of the three values 0,1 , and 2 in this case. Then the measure corresponding to $\left|s_{3}\right\rangle \otimes\left|s_{3}\right\rangle$ is

$$
\frac{1}{6}\left|\epsilon_{i_{11} i_{21} i_{31}} \epsilon_{i_{12} i_{22} i_{32}} c_{i_{11} i_{12}} c_{i_{21} i_{22}} c_{i_{31} i_{32}}\right| .
$$

This is equal to $|\operatorname{det} C|$, where $C \equiv\left\{c_{i j}\right\}$ is a $3 \times 3$ matrix. It is obvious how to generalize this measure to a $N \times N$ system.

In general, a singlet representation of $\mathrm{SU}(N)$ can be constructed from $k N$ defining representations, where $k \geq 1$ is an integer. For $k=1$, the completely antisymmetric representation is the only singlet. There are many singlets for $k \geq 2$. For example, there are three sixth order singlets for $\mathrm{SU}(3)$.

\section{NONSINGLET REPRESENTATIONS}

A representation whose highest weight is neither "maximum" nor "minimum" (i.e., $\left.\boldsymbol{\nu}_{\alpha} \neq q \boldsymbol{e}, \mathbf{0}\right)$ also seems useful to classify entanglement, since the projection thereto vanishes for nonentangled states. We do not know if its norm (or some function thereof) is an entanglement monotone or not. We cannot apply our previous proof of monotonicity in this case because the SLOCC group does not preserve the norm and hence $\left.\left|P_{\boldsymbol{\nu}_{\alpha}}\right| \psi^{\otimes q}\right\rangle \mid$ is not an invariant of that group unless $\boldsymbol{\nu}_{\alpha}=0$. We can, however, show that if $P_{\boldsymbol{\nu}_{\alpha}}\left|\psi^{\otimes q}\right\rangle \neq 0$ and $P_{\nu_{\alpha}}\left|\phi^{\otimes q}\right\rangle=0,|\psi\rangle$ and $|\phi\rangle$ does not belong to the same SLOCC class. The point is that an irrep $R_{\nu_{\alpha}}$ of the LU group is also an irrep of the SLOCC group, since the Lie algebra of the SLOCC group is the complexification of the Lie algebra of the LU group. Therefore $g P_{\boldsymbol{\nu}_{\alpha}}\left|\psi^{\otimes q}\right\rangle=P_{\boldsymbol{\nu}_{\alpha}}(g|\psi\rangle)^{\otimes q} \in R_{\boldsymbol{\nu}_{\alpha}}$ for any $g$ in the SLOCC group, and if $P_{\nu_{\alpha}}\left|\psi^{\otimes q}\right\rangle \neq 0$, $P_{\nu_{\alpha}}(g|\psi\rangle)^{\otimes q} \neq 0$ because $g$ is invertible. Hence there is no $g$ in the SLOCC group such that $g|\psi\rangle=|\phi\rangle$.

Let us consider two copies of a state in a $N_{1} \times \cdots \times N_{m}$ multipartite system as an example. Two copies of a defining representation of $\mathrm{SU}\left(N_{i}\right)$ is decomposed into symmetric and antisymmetric irreducible components. We denote the projection operators for the symmetric and antisymmetric components by $P_{+, i}$ and $P_{-, i}$, respectively. The norm of an irre- 
ducible component $\left.\left|P_{s_{1}, 1} \otimes \cdots \otimes P_{s_{m}, m}\right| \psi^{\otimes 2}\right\rangle \mid\left(s_{j}= \pm\right)$ is the generalized concurrence defined in [4]. For example, for the 3-qubit case [3]

$$
\left.\left|P_{-, 1} \otimes P_{-, 2} \otimes P_{+, 3}\right| \psi^{\otimes 2}\right\rangle\left.\right|^{2}=\frac{1}{4} C_{A B}^{2}+\frac{1}{8} \tau_{3} .
$$

Here, $C_{A B}$ is the concurrence [13] for the first and the second qubits.

\section{EXPERIMENTAL OBSERVATION}

Since an irrep of the LU group can be written in the product form $R_{\nu_{1}} \otimes \cdots \otimes R_{\nu_{m}}$, the projection operator thereto can also be written as the tensor product of local projection operators $P_{\nu}=P_{\nu_{1}} \otimes \cdots \otimes P_{\nu_{m}}[14]$. Therefore the value of our measure can be determined by local projective measurements of some copies of the state. Note that a recently proposed scheme for experimental determination of the SLOCC class of a 3-qubit state [15] is easily obtained from our method using Eqs. (35) and (40).

\section{CONCLUSION AND REMARKS}

In this paper, we have considered irreducible decomposition of $q$ copies of a quantum state $\left|\psi^{\otimes q}\right\rangle$ with respect to the LU group, and shown that the norm of the projection to an irreducible component can be a good measure of entanglement. The irreps can be classified into three types: (A) irrep with the "maximum" highest weight, (B) irrep with the "minimum" highest weight (singlet), (C) others. If the state $|\psi\rangle$ is unentangled, $\left|\psi^{\otimes q}\right\rangle$ is in the type A irrep. Therefore projections to type $\mathrm{B}$ and type $\mathrm{C}$ components vanish. We have shown that an entanglement monotone is obtained from the projection to a type B component. We have also shown that the projection to a type $\mathrm{C}$ component is useful for SLOCC classification of entanglement.

A remaining problem of our method is that independent irreducible components do not necessarily give independent measures. For example, there are many (actually $2^{3}=8$ ) independent fourth order singlets in the 3-qubit case, but the 3-tangle is the only measure obtained from them. It happens because we do not take into account the permutation symmetry of the copies of a quantum state; since $\left|\psi^{\otimes q}\right\rangle$ is completely symmetric with respect to permutations of the copies, only completely symmetric irreducible components survive after projection. It means that we should consider irreducible decomposition not only under the local unitary group, but also under the permutation group of the copies. Progress in this direction will be reported in the forthcoming paper [16]. The permutation group of the parties also would be useful. We hope extensive use of the group theory would provide insight into the nature of multipartite entanglement.

Another important problem is to generalize our method from pure to mixed states. We can formally define an entanglement measure for mixed states $E(\rho)$ from that for pure states $E(|\psi\rangle)$ via the convex roof construction $[17,18]$

$$
E(\rho)=\min \sum_{i} p_{i} E\left(\left|\psi_{i}\right\rangle\right), \quad \rho=\sum_{i} p_{i}\left|\psi_{i}\right\rangle\left\langle\psi_{i}\right| .
$$

Although it is in general difficult to find the set $\left\{p_{i}\right\}$ which gives the minimum value, the group theoretical nature of our method and the projection operator form of the measures could help in solving this problem.

\section{ACKNOWLEDGMENT}

The author would like to thank A. Miyake for helpful comments.
[1] W. Dür, G. Vidal, and J. I. Cirac, Phys. Rev. A 62, 062314 (2000).

[2] A. Miyake, Phys. Rev. A 67, 012108 (2003).

[3] A. Sugita, J. Phys. A 36, 9081 (2003).

[4] F. Mintert, M. Kuś, and A. Buchleitner, Phys. Rev. Lett. 95, 260502 (2005).

[5] A. Osterloh and J. Siewert, Phys. Rev. A 72, 012337 (2005); Int. J. Quantum Inf. 4, 531 (2006).

[6] The first and the second order "combs" in [6] correspond to $\left|s_{2}\right\rangle$ and $\left|s_{4, a}\right\rangle$, respectively, in this paper.

[7] H. Georgi, Lie Algebras in Particle Physics (Benjamin/ Cumming, Reading, MA, 1982).

[8] A. Sugita, J. Phys. A 35, L621 (2002).

[9] F. Mintert and K. Życzkowski, Phys. Rev. A 69, 022317 (2004).

[10] F. Verstraete, J. Dehaene, and B. De Moor, Phys. Rev. A 68, 012103 (2003).

[11] S. Schenk and G.-L. Ingold, Phys. Rev. A 75, 022328 (2007).
[12] V. Coffman, J. Kundu, and W. K. Wootters, Phys. Rev. A 61, 052306 (2000).

[13] W. K. Wootters, Phys. Rev. Lett. 80, 2245 (1998).

[14] If there is more than one independent singlet of the LU group of a given order, it is possible to make another singlet from a linear combination of the singlets. For example, in the 4-qubit case, we can make a 4th order singlet of the form $\left|s_{4, a}\right\rangle$ $\otimes\left|s_{4, b}\right\rangle \otimes\left|s_{4, b}\right\rangle \otimes\left|s_{4, b}\right\rangle+\left|s_{4, a}\right\rangle \otimes\left|s_{4, a}\right\rangle \otimes\left|s_{4, b}\right\rangle \otimes\left|s_{4, b}\right\rangle$. In such a case, the corresponding projection operator cannot be written as a product of local projection operators. Then we have to combine several different local measurements to determine the value of the corresponding measure.

[15] C. S. Yu and H. S. Song, Phys. Rev. A 76, 022324 (2007).

[16] A. Sugita (unpublished).

[17] C. H. Bennett, D. P. DiVincenzo, J. A. Smolin, and W. K. Wootters, Phys. Rev. A 54, 3824 (1996).

[18] A. Uhlmann, Phys. Rev. A 62, 032307 (2000). 